\title{
CORRECTION
}

\section{Author Correction: Targeting Hsp90 with FS-108 circumvents gefitinib resistance in EGFR mutant non-small cell lung cancer cells}

Yue-qin Wang ${ }^{1}$, Ai-jun Shen ${ }^{2}$, Jing-ya Sun ${ }^{2}$, Xin Wang ${ }^{2}$, Hong-chun Liu ${ }^{2}$, Min-min Zhang ${ }^{2}$, Dan-qi Chen ${ }^{3}$, Bing Xiong ${ }^{3}$, Jing-kang Shen ${ }^{3}$, Mei-yu Geng ${ }^{2}$, Min Zheng ${ }^{4}$ and Jian Ding ${ }^{2}$

Acta Pharmacologica Sinica (2020) 41:731-732; https://doi.org/10.1038/s41401-019-0341-6

Correction to: Acta Pharmacol Sin https://doi.org/10.1038/ aps.2016.85, published online 12 Sep 2016

It just came to the author's attention that three errors were introduced into the paper during the stage of figure preparation.

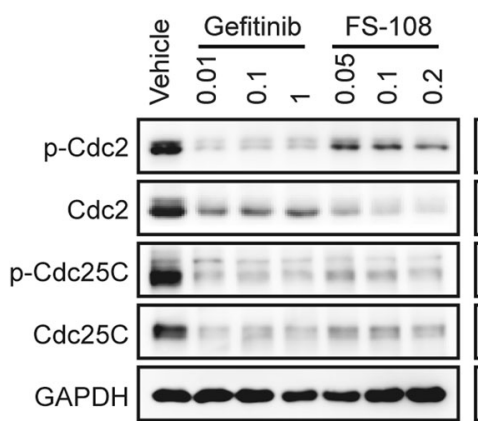

HCC 827

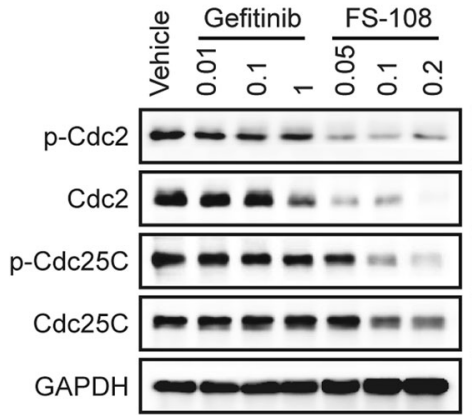

$\mathrm{NCl}-\mathrm{H} 1650$

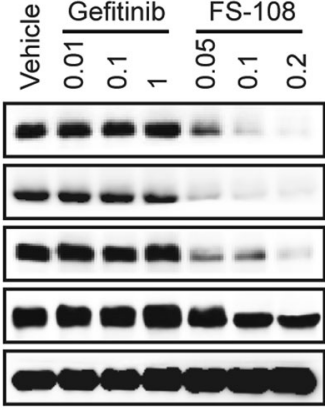

HCC827/GR6

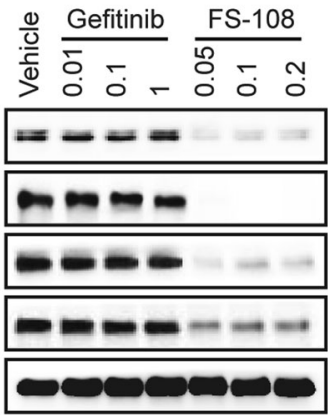

$\mathrm{NCl}-\mathrm{H} 1975$
Fig. 2b Impact of FS-108 on $\mathrm{G}_{2} / \mathrm{M}$ transition regulators. HCC827, $\mathrm{HCC} 827 / \mathrm{GR6}, \mathrm{NCl}-\mathrm{H} 1650$, and $\mathrm{NCl}-\mathrm{H} 1975$ cells were treated with gefitinib or FS-108 at the indicated concentrations for $24 \mathrm{~h}$ and the cell lysates were immunoblotted with the indicated antibodies.
Specifically, the Western blotting bands of GAPDH in Fig. $2 b$ as well as Fig. 3b, cv PARP in HCC827/GR6 cells (Fig. 3b) and IHC staining images of EGFR and Hsp70 in group gefitinib $50 \mathrm{mg} / \mathrm{kg}$ (Fig. 5d) were found to be misplaced inadvertently. These errors

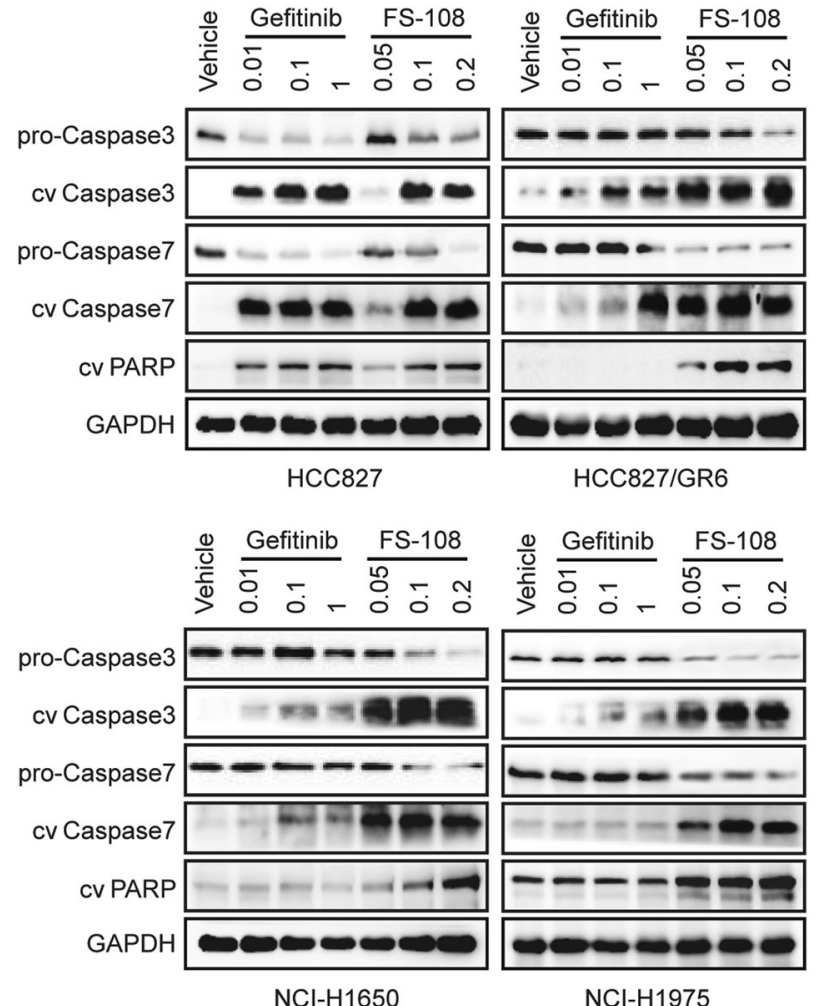

Fig. 3b Modulation of FS-108 on apoptotic proteins. HCC827, $\mathrm{HCC} 827 / \mathrm{GR6}, \mathrm{NCl}-\mathrm{H} 1650$, and $\mathrm{NCl}-\mathrm{H} 1975$ cells were treated with gefitinib or FS-108 at the indicated concentrations for $72 \mathrm{~h}$ and analyzed by immunoblotting with the indicated antibodies.

\footnotetext{
'Department of New Drug Screening Center, China Pharmaceutical University, Nanjing 210009, China; ${ }^{2}$ Division of Anti-tumor Pharmacology, Synthetic Organic and Medicinal Chemistry Laboratory, State Key Laboratory of Drug Research, Shanghai Institute of Materia Medica, Chinese Academy of Sciences, Shanghai 201203, China; ${ }^{3}$ Synthetic Organic and Medicinal Chemistry Laboratory, State Key Laboratory of Drug Research, Shanghai Institute of Materia Medica, Chinese Academy of Sciences, Shanghai 201203, China and ${ }^{4}$ Department of Thoracic Surgery, Shanghai Tongren Hospital, Shanghai Jiao Tong University School of Medicine, Shanghai 200336, China

Correspondence: Jian Ding (jding@simm.ac.cn) or Min Zheng (zhengmin8010@126.com) or Mei-yu Geng (mygeng@simm.ac.cn)

These authors contributed equally: Yue-qin Wang, Ai-jun Shen
}

Published online: 21 February 2020 
Author Correction: Targeting Hsp90 with FS-108 circumvents gefitinib...

Yue-qin Wang et al.

732

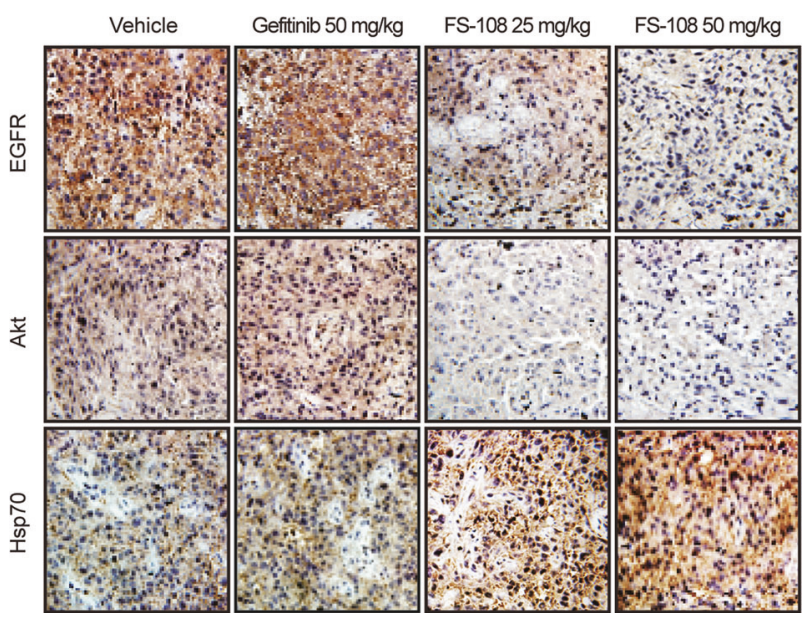

Fig. 5d Effects of FS-108 on the modulation of protein expression in vivo. The immunohistochemical evaluation of the basal expression of EGFR, Akt, and Hsp70 is presented. were made completely by accident. Under no circumstance did the authors falsify or "make up" data in order to mislead the reviewers or readers.

These corrections do not affect the scientific conclusions of each section and the entire paper. Nonetheless, the authors sincerely apologize for any inconvenience this might have caused.

The corrected figure panels of Fig. $2 \mathrm{~b}, 3 \mathrm{~b}$ and $5 \mathrm{~d}$ are presented as follows: 